\title{
Sub-wavelength imaging with a left-handed material flat lens
}

\author{
Michael W. Feise and Yuri S. Kivshar \\ Nonlinear Physics Group and Centre for Ultra-high bandwidth Devices for Optical Systems (CUDOS), \\ Research School of Physical Sciences and Engineering, \\ Australian National University, Canberra, ACT 0200, Australia
}

(Dated: November 13, 2018)

\begin{abstract}
We study numerically, by means of the pseudospectral time-domain method, the unique features of imaging by a flat lens made of a left-handed metamaterial that possesses the property of negative refraction. We confirm the earlier finding that a left-handed flat lens can provide near-perfect imaging of a point source and a pair of point sources with clear evidence of the sub-wavelength resolution. We illustrate the limitation of the resolution in the time-integrated image due to the presence of surface waves.
\end{abstract}

PACS numbers: 42.30.Wb, 78.20.Ci, 42.25.Bs, 41.20.Jb

Keywords: Left-handed material, double-negative material, negative refractive index, image resolution, surface waves

The unique properties of left-handed (LH) materials [1], i.e. materials with simultaneously negative real parts of the dielectric permittivity $\epsilon_{r}$ and the magnetic permeability $\mu_{r}$ (which can also be described by a negative index of refraction), allow focusing of electromagnetic waves by a flat slab of the material; this effect is in sharp contrast to conventional optical lenses with a positive refractive index that need to have curved surfaces to form an image. Recently, Pendry [2] argued that a slab of a lossless LH material with $\epsilon_{r}=\mu_{r}=-1$ should behave as a perfect lens enabling one to obtain an ideal image of a point source through the reconstitution of the evanescent wave components. While recent experiments confirmed the main features of negative refraction [3, 4], the question of near-perfect imaging of a flat lens and near-field focusing has remained highly controversial [5], and is severely constrained because of large dissipation and anisotropy in the metamaterial. Nevertheless, several studies showed that nearly-perfect imaging should be expected even under realistic conditions when both dispersion and losses of the left-handed material are taken into account [6, 7, , 8, 9, 10].

In this Letter, we re-visit the problem of nearly perfect imaging by a flat lens made of a left-handed metamaterial and study numerically, by use of the pseudospectral time-domain method, imaging by a flat lens made of this material. In order to study the amplification of the evanescent waves, we compare the wave-vector spectra of the field at the image plane in the case with and without the LH slab. We confirm the earlier finding that a left-handed flat lens can provide near-perfect imaging of a point source [7] and a pair of point sources with clear evidence of sub-wavelength resolution. In addition we consider the time-resolved and time-integrated Poynting vector at the source frequency which behaves equivalently to the time-integrated intensity that may be relevant in lithography applications.

We model an electrical line current source in front of a slab of LH material embedded in free space, as shown in Fig. 1. The system is translationally invariant in the

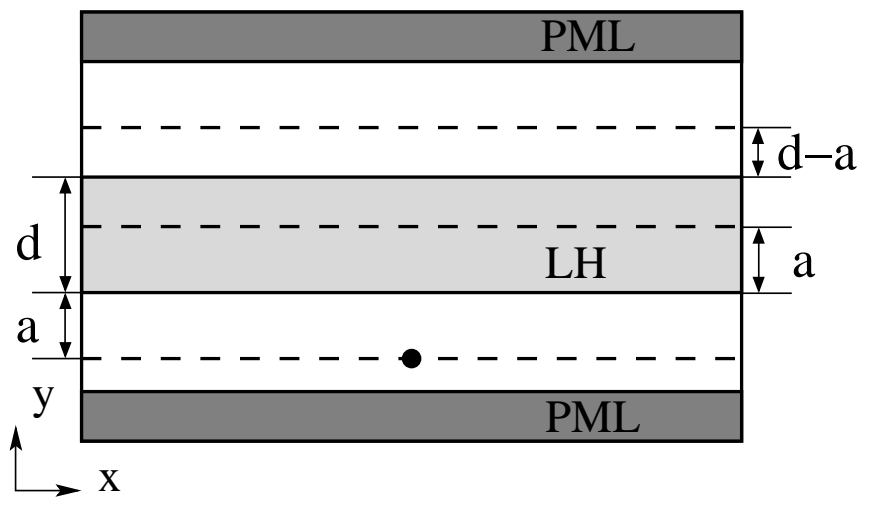

FIG. 1: Schematic view of the model system. A current source is placed in front of a LH slab. In $y$ direction the system models an open domain through the use of reflectionless absorbing boundaries (PML), while it is periodic in the $x$ direction. The solid dot indicates the source location while the dashed lines denote the observation planes, i.e. the source and image planes.

$z$ direction and is treated as two-dimensional. The simulations are performed in TE polarization $\left(H_{x}, H_{y}, E_{z} \neq\right.$ $0)$. In the $y$ direction the system is considered open, as achieved through reflectionless absorbing boundaries, while the $x$ direction is taken as periodic. Because of this the simulation essentially uses an infinite slab and an array of sources.

It has been shown that a material with left-handed character can only be achieved through dispersion [1]. Therefore, the LH material is assumed to have lossy Drude characteristics in both electric permittivity and magnetic permeability [2], given by

$$
\begin{aligned}
& \varepsilon_{r}(\omega)=1-\frac{\omega_{p e}^{2}}{\omega\left(\omega+i \gamma_{e}\right)}, \\
& \mu_{r}(\omega)=1-\frac{\omega_{p m}^{2}}{\omega\left(\omega+i \gamma_{m}\right)} .
\end{aligned}
$$

Here $\omega_{p e}, \omega_{p m}$ are the plasma frequencies and $\gamma_{e}, \gamma_{m}$ 
are the collision frequencies of the electric and magnetic properties, respectively. To simplify and to impedance match the slab to free space, we take $\omega_{p e}=\omega_{p m}=\omega_{p}$ and $\gamma_{e}=\gamma_{m}=\gamma$. The material parameters are chosen to give a refractive index with real part $\operatorname{Re}(n)=-1$ at frequency $f_{0}=15 \mathrm{GHz}\left(\omega_{0}=2 \pi f_{0}\right)$. For this we use $\omega_{p}=2 \pi \sqrt{2} f_{0}$. The collision frequency is $\gamma=2 \pi \times 4.5 \mathrm{MHz}$, which results in $\varepsilon_{r}\left(\omega_{0}\right)=\mu_{r}\left(\omega_{0}\right)=-1+0.0006 i$.

We directly simulate the field propagation based on Maxwell's equations, using the pseudospectral timedomain method [1]. In this method all field components are sampled at the same spatial location which avoids ambiguities of the material interfaces [12] inherent in the usual finite-difference time-domain method [13, 14], and the problems caused by transition layers at the interfaces [15. The domain walls in the $y$ direction are covered with a uniaxial perfectly-matched layer (PML) 16, 17, 18] boundary to simulate an open domain. In the $x$ direction the system is periodic. The material constitutive relations are implemented using the auxiliary differential equation method [19].

The current source is turned on slowly using a temporal dependence $\left(1-e^{-t / \tau}\right) \sin \left(\omega_{0} t\right)$, with a turn-on parameter $\tau \approx 22 / f_{0}$, to reduce its band width and allow the system to reach steady-state faster.

The simulation uses a time step of $\Delta_{t}=29.2873 \mathrm{~ns}$ and a spatial step size of $\Delta_{x}=\Delta_{y}=\lambda_{0} / 100=0.199862 \mathrm{~m}$; $\lambda_{0}$ is the free-space propagating wavelength at frequency $f_{0}$. The simulation size is $1024 \times 128$ and is iterated for 600000 time steps, i.e. 2635 periods, to ensure steadystate.

It has been shown that a slab of LH material with parallel sides should focus propagating [1] and evanescent 2] waves. Thus, if the slab thickness $d$ is greater than the distance $a$ between the object (source) and the front face of the slab, then one expects an image to form inside the slab, a distance $a$ from the front face, as well as behind the slab, a distance $d-a$ from the back face, as indicated in Fig. 1

First, we place a single source a distance $a=0.2 \lambda_{0}$ in front of a slab with thickness $\lambda_{0} / 3$. Snapshots of the electric field in the source plane and the two image planes are shown in Fig. 22 The snapshots were taken when the field was at its maximum in the source plane, which was also the time of maximum field in the image planes. The field of the source plane is well reproduced in the image planes. The feature size of the central peak in the image planes is well below the free-space limit of $\lambda_{0} / 2$. To illustrate that point we performed a spatial Fourier transform of this data and show the transverse spatial frequency dependence of the image-plane fields relative to the source-plane field in Fig. 3. For comparison the figure also shows the spectrum in the same planes when the LH slab is replaced by air. In the case without the LH slab, waves with spatial frequency greater than $1 / \lambda_{0}$, corresponding to evanescent waves, are almost entirely absent in the image planes. In contrast, when the LH slab is present, waves with spatial frequency up to $3.5 / \lambda_{0}$

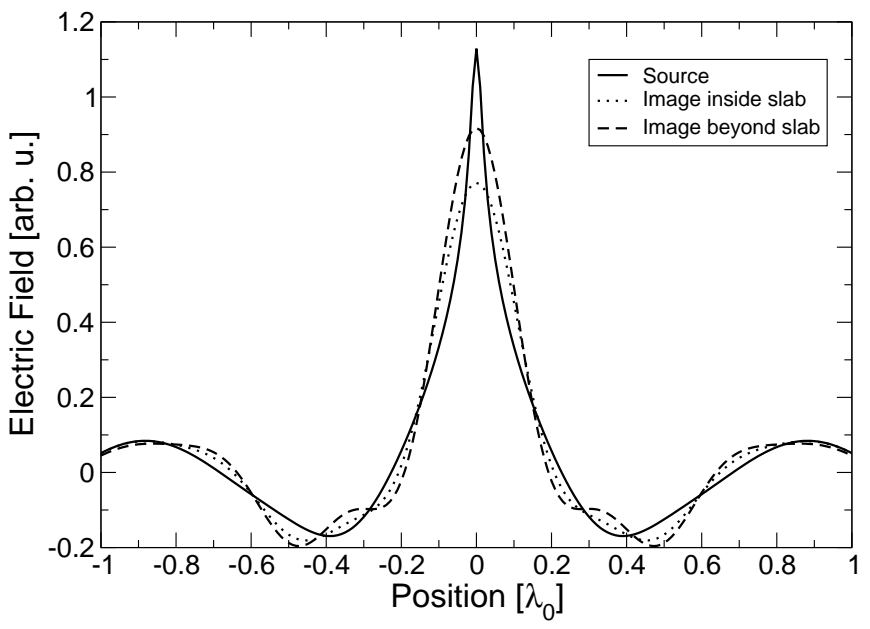

FIG. 2: Snapshots of the electric field in the source plane (solid), the image plane inside the slab (dotted) and beyond the slab (dashed) with a single source.

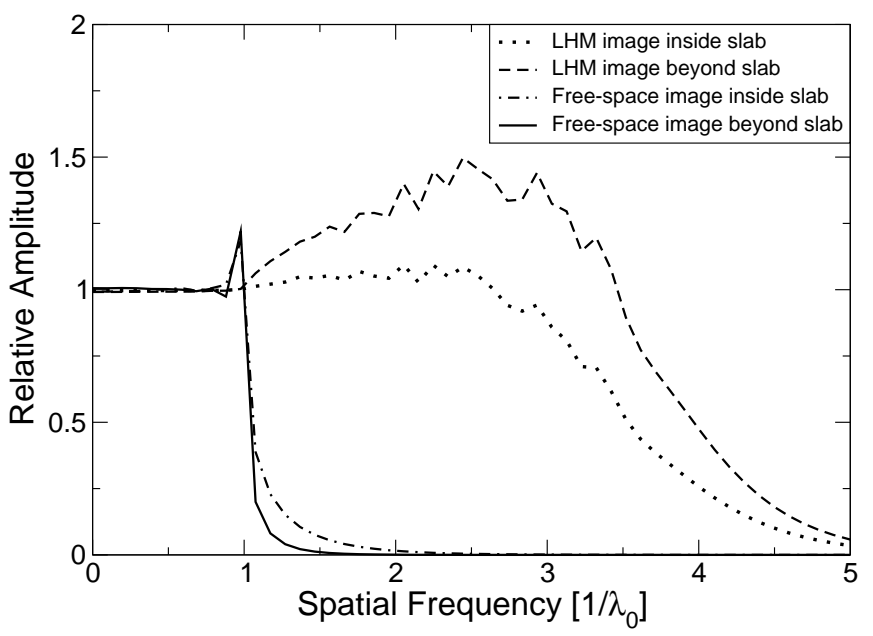

FIG. 3: Transverse wave vector dependence of the fields in the image planes relative to the field in the source plane in the case of a single source; with LH slab: image location inside slab (dotted), image location beyond slab (dashed); free-space: image location inside slab (dash-dotted), image location beyond slab (solid).

are transmitted to the image locations. This agrees with the smaller than $\lambda_{0} / 2$ features present in Fig. 2 The wave vector cutoff value $\left(k_{c}=2 \pi \lambda_{c}\right)$ seen in Fig. 3 is comparable to the value expected according to the parameters used in the system [8]. At $2.8 / \lambda_{0}$ a peak occurs in the spectrum, similar to the results reported in [20].

We also study the imaging of a pair of sources in order to characterize the possibility of sub-wavelength resolution. The single source is replaced by a pair, separated by $0.34 \lambda_{0}$. The two sources have the same time dependence, i.e., are in-phase. In Fig. 目 snapshots of the $y$ component of the Poynting vector $\left(S_{y}\right)$ in the image plane beyond the slab are shown for two different slab thicknesses while the distance between the sources and the 


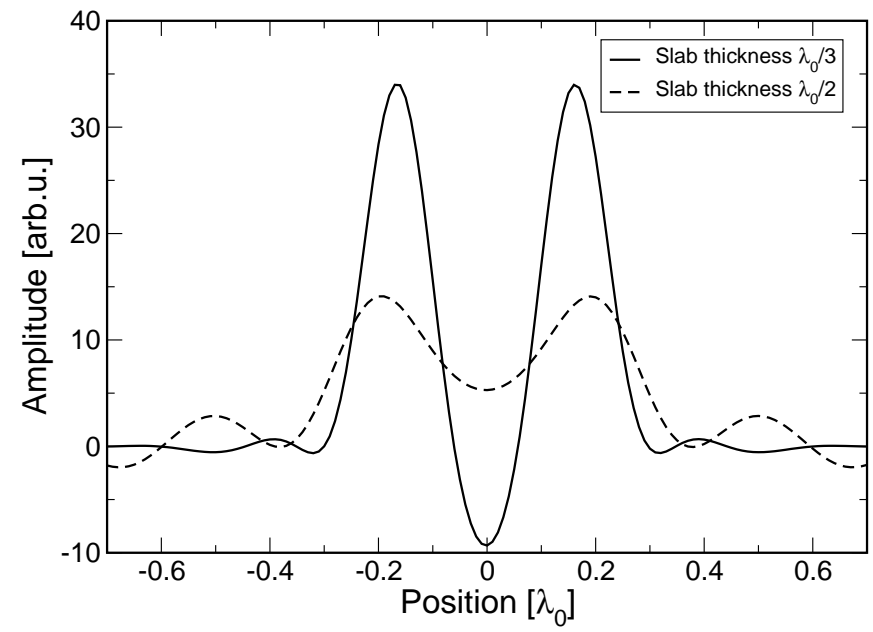

FIG. 4: Snapshot of the $y$ component of the Poynting vector in the image plane beyond the slab for a slab thickness of $\lambda_{0} / 3$ (solid) and $\lambda_{0} / 2$ (dashed).

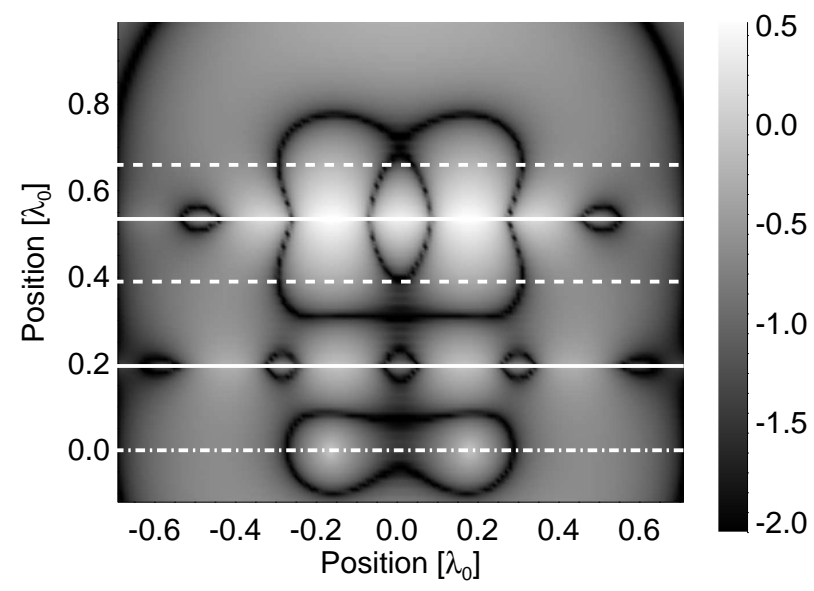

FIG. 5: Absolute magnitude of the electric field on a logarithmic scale at time $t=2635 / f_{0}$ with two sources in front of the LH slab of thickness $\lambda_{0} / 3$. The LHM slab is denoted by solid lines, the image planes by dashed lines, and the source plane by a dash-dotted line.

slab is keept constant. The snapshots are again taken at the same time and show the instance with highest image peaks. One finds that features with sizes less than $\lambda_{0} / 2$ are reproduced in the images but the quality of the sub-wavelength resolution diminishes with increased slab thickness [8].

With slab thickness $\lambda_{0} / 3, S_{y}$ in the region between the image peaks has opposite sign compared to that of the image peaks. This is a signature of the influence of the surface waves on the image.

Figure 5]shows a two-dimensional false-color plot of the absolute magnitude of the electric field with two sources on a logarithmic scale. The geometry and orientation is as indicated in Fig. 1] One clearly sees the two sources and the strong fields they excite at the surfaces. The

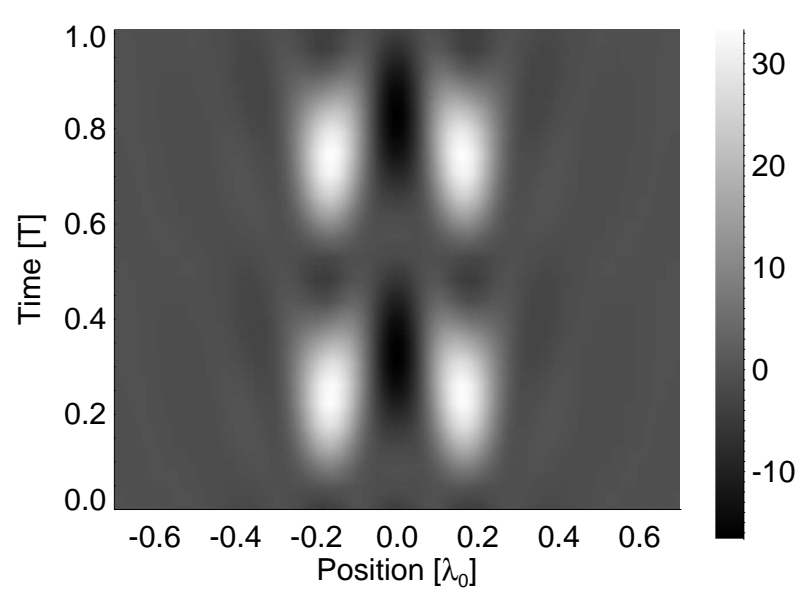

FIG. 6: Time dependence of the amplitude of the $y$ component of the Poyting vector $\left(S_{y}\right)$ in the image plane beyond a slab of thickness $\lambda_{0} / 3$ on a linear scale.

surface waves rapidly diminish within $\lambda_{0} / 2$ on either side of the source-image axis. One notices that in the image planes the source images are resolved in the transverse, i.e. $x$, direction but not in the $y$ direction. Again, we see the additional peak between the image peaks and extrema of the surface waves to both sides.

To understand the behavior of the surfave waves we studied the transfer function of the system, which is easily found by matching the boundary conditions of the fields at the slab surfaces. With the parameters of the system given above, we found that the appearance of the surface waves is dominated by the high wave vector components contained in the source distribution. These wave vector components lie beyond the maximum wave vector value $k_{c}$ that the slab can image correctly. A source distribution with less contribution beyond $k_{c}$ suffers less distortion in the image from surface waves. The slab thickness and the material parameters determine $k_{c}$ which in turn influences the occurrance of the surface waves.

Unlike the cavity effect observed for a slab of finite width [21], we found that the distance to the neighboring sources (present due to the periodic boundary conditions in $x$ ) has no noticable influence on the image, as long as this distance is at least a few $\lambda_{c}$.

To illustrate the temporal behavior of the image, in Fig. 6] we plot $S_{y}$ in the image plane beyond the slab as a function of time for a slab of thickness $\lambda_{0} / 3$. The image peaks are visible as areas of large positive value. Between the image peaks, with a time delay of about $T / 8$, a negative peak appears. The curve in Fig. 4 corresponds to a slice at time $0.22 T$ in Fig. 6. Upon careful inspection one can see the peaks of the surface waves moving away from the image peaks and then disappear. When the slab thickness is increased to $\lambda_{0} / 2$ (not shown), the region of negative $S_{y}$ has essentially disappeared and become a region of relatively large positive $S_{y}$. Rather than being clearly distinct, the two image peaks begin to merge, as 
can be seen in Fig. 4 The surface waves present have lower $k_{c}$, i.e., larger $\lambda_{c}$, and greater amplitude. They also move faster away from the image peaks.

From Fig. 6] it is evident that the time-integrated Poynting vector has an additional significant extremum between the two image peaks, which will lead to a distortion of the image. In general, this peak due to surface waves is not distinguishable from a genuinely imaged peak in the source plane. This poses an important limitation on the usable parameter range of the left-handed material flat lens.

In conclusion, we have studied the unique features of imaging by a flat lens made of a left-handed metamaterial that possesses the property of negative refraction. By employing the pseudospectral time-domain method and comparing with the wave propagation in air, we have confirmed previous results of near-perfect imaging of a point source and a pair of point sources with clear evidence of sub-wavelength resolution. We found that the time-integrated image has an additional limiting factor on the resolution of the images of multiple close objects due to substantial constructive interference in the image plane. We believe that a potential advantage of this kind of imaging is its scalability to sub-micrometer scales to make possible imaging and nano-photolithography with spatial resolution in the tens of nanometers.

This work has been partially supported by the Australian Research Council and the Australian National University. The authors thank I. V. Shadrivov for helpful collaboration, N. A. Zharova and A. A. Zharov for useful discussion of left-handed materials, as well as S. A. Cummer, J. B. Schneider and R. W. Ziolkowski for useful discussion of numerical methods.
[1] V. G. Veselago, Phys. Usp. 10, 509 (1968).

[2] J. B. Pendry, Phys. Rev. Lett. 85, 3966 (2000), URL http://link.aps.org/abstract/PRL/v85/p3966

[3] R. A. Shelby, D. R. Smith, and S. Schultz, Science 292, 77 (2001).

[4] C. G. Parazzoli, R. B. Greegor, K. Li, B. E. C. Koltenbah, and M. Tanielian, Phys. Rev. Lett. 90, 107401 (2003), URL http://link.aps.org/abstract/PRL/v90/p107401

[5] L. Venema, Nature 420, 119 (2002).

[6] N. Fang and X. Zhang, Appl. Phys. Lett. 82, 161 (2003).

[7] S. A. Cummer, Appl. Phys. Lett. 82, 1503 (2003).

[8] D. R. Smith, D. Schurig, M. Rosenbluth, S. Schultz, S. A. Ramakrishna, and J. B. Pendry, Appl. Phys. Lett. 82, 1506 (2003).

[9] W. T. Lu and S. Sridhar, Microw. Opt. Technol. Lett. 39, 282 (2003).

[10] M. Nieto-Vesperinas, J. Opt. Soc. Am. A 21, 491 (2004).

[11] Q. H. Liu, Microw. Opt. Technol. Lett. 15, 158 (1997).

[12] M. W. Feise, J. B. Schneider, and P. J. Bevelacqua, IEEE Trans. Antennas Propag. 52, 2955 (2004).
[13] K. S. Yee, IEEE Trans. Antennas Propag. AP14, 302 (1966).

[14] A. Taflove and S. C. Hagness, Computational Electrodynamics: The Finite-Difference Time-Domain Method (Artech House, Boston, 2000), 2nd ed., ISBN 1-58053076-1.

[15] M. W. Feise, P. J. Bevelacqua, and J. B. Schneider, Phys. Rev. B 66, 035113 (2002), URL http://link.aps.org/abstract/PRB/v66/p035113

[16] J. P. Berenger, J. Comput. Phys. 114, 185 (1994).

[17] Z. S. Sacks, D. M. Kingsland, R. Lee, and J. F. Lee, IEEE Trans. Antennas Propag. 43, 1460 (1995).

[18] M. Kuzuoglu and R. Mittra, IEEE Microw. Guided Wave Lett. 6, 447 (1996).

[19] R. M. Joseph, S. C. Hagness, and A. Taflove, Opt. Lett. 16, 1412 (1991).

[20] X. S. Rao and C. K. Ong, Phys. Rev. E 68, 067601 (2003).

[21] L. Chen, S. L. He, and L. F. Shen, Phys. Rev. Lett. 92, 107404 (2004). 